\title{
The Space-Time Evolution Rules of Plastic Failure Zone in Surrounding Rock of Stope Roof with Variable Length
}

\author{
Xinfeng Wang ${ }^{1,2, *}$, Longqin $\mathrm{Li}^{2}$ and Jiaqing $\mathrm{Wu}^{2}$ \\ ${ }^{1}$ Hunan Key Laboratory of Geomechanics and Engineering Safty, Xiangtan University, Xiangtan 411105, China \\ ${ }^{2}$ College of Environment and Resources, Xiangtan University, Xiangtan 411105, China \\ ${ }^{*}$ Corresponding author
}

\begin{abstract}
In order to study the forming process, development trend and failure pattern of plastic zone in surrounding rock of stope roof with variable length, the analytical formula of the radius plastic zone, tangential stress and radial stress of surrounding rock were derived by using the limit equilibrium theory of elastic plastic mechanics. Based on the numerical simulation software, the space-time evolution rules of plastic zone gradual destruction in surrounding rock of stope roof with variable length was studied. Research shows that the stability of surrounding rock and the original rock should force, cohesion and internal friction angle is closely related, cutting to stress and radial stress increases with the increase of the cohesion increases, the radius of plastic zone with the cohesion and internal friction angle increases and decreases. Facet mining roof surrounding rock plastic zone formation produced at the initial stage of plastic failure, the development stage of the formation of elliptical partial plastic ring, extended late in the formation of "cyclic damage ring”. Large mining roof surrounding rock plastic zone expansion of space from the shallow to the deep extension transfer, damage range from the lower part to upper part of the vertical extension, the plastic zone shape by circular circle development is the knife shaped and exhibited the gradual failure characteristics under the time and space condition.
\end{abstract}

Keywords-stope with variable length; roof surrounding rock; plastic failure zone; evolution rules

\section{INTRODUCTION}

Due to the mass exploitation of coal resources, geological condition is superior, simple geological environment, coal seam buried depth is shallow, roadway design and construction management difficulty of small mine gradually reduced, coal mining into deep extension, long face mutation increased, the deterioration of the geological conditions, rock structure of the alienation of complicated mining stage. Stope with variable length in the period of rapid development of coal mining bred and, with increasing of coal mining intensity increases and the difficulty of exploitation, change long working face in coal seam exploitation of the phenomenon is increasing, in quite a long period of time has universality and necessity of widespread. In this paper, the research of stope with variable length mainly refers to the stope mutant, namely in coal mining by engineering geology, fault, joint fold and hydrological gas and geotemperature geopressure, mining environment factors, the length of working face a sudden increase in the "Dao ba shi" mining industry and trade for surface[1-2]. Stope with variable length due to the variation in the length of the sudden and span caused the roof rock rupture shape is irregular, overlying strata spatial structure shows a nonlinear extended situation, dynamic response of dynamic recovery appear more active, surrounding rock plastic zone damage and extend the space with different from the formal mining field progressive spatial and temporal evolution characteristics.

The study shows that the shape, size and range of the plastic zone of surrounding rock is an important index to evaluate the stability and integrity of rock mass. For a long time, domestic and foreign mining scholars have carried out a lot of research work on the destruction range and distribution law of surrounding rock plastic zone. Chen Liwei[3] using unified strength theory is given for the non uniform stress field of roadway surrounding rock plastic zone boundary line equa-tion, and of different lateral pressure coefficients of tunnel surrounding rock plastic zone radius and shape of the effective prediction. Ma Nianjie[4] based on elastic plastic theory of borehole stress and deviator resolution, using the Mohr Coulomb strength criterion the non-uniform stress field of circular tunnel surrounding rock plastic zone spreading radius and butterfly distribution. Zhang Changguang[5] based on the comprehensive consideration of elastic strain, brittle softening and expansion minus weakening and plastic deformation of roadway surrounding rock, introducing intermediate principal stress force law established the roadway surrounding rock plastic zone of strain and displacement should be analytical solution of stress. With the help of the elastic plastic mechanical properties of limit equilibrium theory, deduced the surface long mining field of surrounding rock plastic zone radius, cut to stress and radial should be analytical solution by using the numerical simulation technology of roof surrounding rock plastic zone damage range and the expansion of the trend, reveal the surface long mining roof surrounding rock elastic plastic damage evolution process.

\section{Mechanical Analysis of Plastic Zone in} SURROUNDING ROCK OF STOPE ROOF WITH VARIABLE LENGTH

The surrounding rock is assumed to be a circular hole structure, and the surrounding rock is in a state of hydrostatic pressure, and the equilibrium equation of the limit equilibrium 
zone of surrounding rock is constructed by using the limit equilibrium theory[6].

$$
r \frac{d \sigma_{r}}{d r}+\sigma_{r}-\sigma_{t}=0
$$

Using the limit equilibrium condition

$$
\sigma_{t}=\frac{1+\sin \varphi}{1-\sin \varphi} \sigma_{r}+\frac{2 C \cos \varphi}{1-\sin \varphi}
$$

Taking formula (2) into formula (1)

$$
r \frac{d \sigma_{r}}{d r}+\sigma_{r}-\frac{1+\sin \varphi}{1-\sin \varphi} \sigma_{r}-\frac{2 C \cos \varphi}{1-\sin \varphi}=0
$$

After the formula (3) transformation

$$
\frac{d \sigma_{r}}{\sigma_{r}+C \cot \varphi}=\frac{2 \sin \varphi}{1-\sin \varphi} \times \frac{d r}{r}
$$

After the formula (4) integral transform

$$
\ln \left(\sigma_{r}+C \cot \varphi\right)=\frac{2 \sin \varphi}{1-\sin \varphi} \ln r+\ln A
$$

Taking formula (5) for a slight improvement

$$
\sigma_{r}+C \cot \varphi=A r^{\frac{2 \sin \varphi}{1-\sin \varphi}}
$$

If $\sigma_{r}=0, r=r_{0}$, then

$$
A=C \cot \varphi / r_{0}^{\frac{2 \sin \varphi}{1-\sin \varphi}}
$$

Then get

$$
\begin{gathered}
\sigma_{r}=C \cot \varphi\left[\left(\frac{r}{r_{0}}\right)^{\frac{2 \sin \varphi}{1-\sin \varphi}}-1\right] \\
\sigma_{t}=C \cot \varphi\left[\frac{1+\sin \varphi}{1-\sin \varphi}\left(\frac{r}{r_{0}}\right)^{\frac{2 \sin \varphi}{1-\sin \varphi}}-1\right]
\end{gathered}
$$

Taking into account the stress environment of surrounding rock is the hydrostatic pressure field, the rock mass in the plastic zone of the surrounding rock is also satisfied with the $\sigma_{r}+\sigma_{t}=2 \sigma_{1}$, take $\sigma_{1}=\lambda H$, then

$$
\operatorname{Ccot} \varphi\left[\frac{1+\sin \varphi}{1-\sin \varphi}\left(\frac{R}{r_{0}}\right)^{\frac{2 \sin \varphi}{1-\sin \varphi}}-1\right]+C \cot \varphi\left[\left(\frac{R}{r_{0}}\right)^{\frac{2 \sin \varphi}{1-\sin \varphi}}-1\right]=2 \gamma H^{(10)}
$$

Seek to obtain

$$
R=r_{0}\left[\frac{(\gamma H+C \cot \varphi)(1-\sin \varphi)}{C \cot \varphi}\right]^{\frac{1-\sin \varphi}{2 \sin \varphi}}
$$

By the analytical formula of the radius of the two way stress and plastic zone in the limit equilibrium zone of the surrounding rock, the stability of surrounding rock is closely related to the original stress, cohesion and internal friction angle. Tangential stress and radial stress were positively correlated with cohesion, which increased with the increase of cohesive force, and the radius of plastic zone is negatively correlated with cohesion and internal friction angle, and decreased with the increase of cohesion and internal friction angle.

\section{NumERICAL SimUlation OF SPACE-Time EVOlUTION Rules of Plastic FAILURE ZONE IN SURROUNDING ROCK OF STOPE ROOF WITH VARIABLE LENGTH}

\section{A. Establish Numerical Model}

According to the specific engineering geological conditions, the theoretical analysis model is established to calculate the deformation instability characteristics and plastic failure process of roof surrounding rock during coal seam mining. The geometric dimensions of the model are as follows: length $\times$ width $\times$ height $=700 \times 400 \times 160(\mathrm{~m})$, a total of $70 \times 40 \times 40$ grid nodes. Stope with variable length has designed for working length from small to large mutant stope of "Dao ba shi". Working face length is $100 \mathrm{~m} \sim 200 \mathrm{~m}$, strike length is $600 \mathrm{~m}$. strike length of $100 \mathrm{~m}$ working face length is $240 \mathrm{~m}$, convergence of $200 \mathrm{~m}$ big face whose strike length is $360 \mathrm{~m}$.Mining model of three to six were set constraints, model around surrounded by fixed horizontal displacement and vertical displacement, the model bottom surface set up force constraint conditions and the strain and displacement constraints, top design for free surface, displacement and stress are not constrained, and above the top of the model was applied for the quantitative of uniformly distributed load.

\section{B. Simulation Result Analysis}

Underground coal mining will inevitably lead to the original rock structure of the second adjustment, resulting in changes in the mechanical properties of rock masses, 
accompanied by elastic energy sudden release and repeat agglomeration, in inner rock cracks and continuous expansion and development, caused the roof rock instability unloading, elastic expansion, plastic deformation and dilatancy damage. The development and expansion of the plastic zone of surrounding rock in the roof rock during the mining of working face with variable length is simulated by computer, the failure mechanism and deformation evolutionary law of surrounding rock in stope roof with variable face length is obtained.
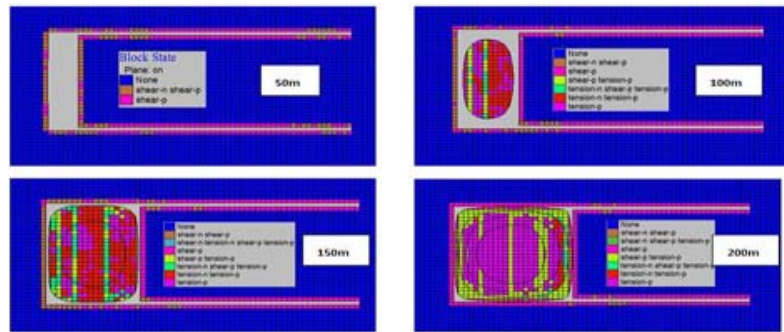

FIGURE I. EXPANSION PROGRESS OF PLASTIC ZONE IN SURROUNDING ROCK OF SMALL STOPE

1) Expansion law of plastic zone in surrounding rock of small stope:The forming process, development trend and space destruction pattern of small stope roof rock plastic zone during the mining period are shown in Figure 1. As can be seen from Figure I, with the increase of the working face advancing distance, the plastic zone of the surrounding rock of stope roof is expanding, the influence range increases, and the extension is obvious. Working face cut eye advance to $50 \mathrm{~m}$, roof surrounding rock plastic zone only around the roof of the formation and development, expansion space is still confined to the long side and a short side and long edge damage prior to the short side, long side were heavily damaged, the surrounding rock produced a small amount of plastic deformation and the most part of the surrounding rock is still in elastic stage. Working face advancing to the $100 \mathrm{~m}$, the roof in the middle of the plastic zone began to appear, the expanding scope of roof around to the top center, and an oval is central to the external radiation extension, surrounding rock by the dual role of shrinkage crack of shear slip and tension. Working face has continued to advance to $150 \mathrm{~m}$ surrounding rock elastic area expansion space to further expand, plastic damage range from ellipse expansion evolution for nearly circular expansion, central roof formed a circle damaged zone ", extend the space from the inside out, gradually regular shape evolution. Again with increase of mining working face and pushed the progress, working face advance to $200 \mathrm{~m}$, promote the length and the length of working face just quite, "square collapse effect" began to highlight, overburden rock activity is frequent, severe damage of brittle rock, sudden release of energy of rock mass surrounding rock elastic zone extending scope extends to the whole panel, the middle part of the top plate formed the progressive, ring white ring destroy circle ", plastic failure area presented non uniform gradient extended state.

Throughout the facet of stope during the mining of surrounding rock plastic zone evolution trend can be found, the roof surrounding rock plastic zone formation, development and expansion with advance increase experienced "long edge of the roof-short side-central space evolution”. Plastic zone formation produced at the initial stage of plastic failure point, development stage of the formation of elliptical partial plastic ring and expansion in the late forming "ring destroy circle", plastic deformation area of influence is gradually expanding, expanding space homeopathic gradual evolution.
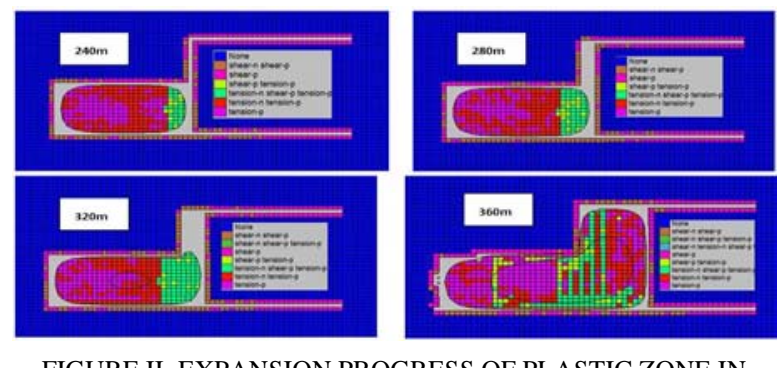

FIGURE II. EXPANSION PROGRESS OF PLASTIC ZONE IN SURROUNDING ROCK OF LARGE STOPE

2) Expansion law of plastic zone in surrounding rock of large stope:After facet mining, mining transition to large mining stage, figure II flects the the surface of working field $240 \mathrm{~m}$ advance to $360 \mathrm{~m}$ during, roof surrounding rock plastic area in the initial formation and medium-term development and full damage morphology evolution. According to figure II ows that, changing the face long mining field by the facet mining into large mining face, surrounding rock plastic zone destruction of space and the expansion of the trend growth significantly, the damage of rock mass both effect of shear and tensile splitting effect control of surrounding rock plastic zone shows a nonli-near discontinuous expansion characteristics. Working face advancing to the $240 \mathrm{~m}$, mining space has just entered joint of surrounding rock elastic the influence degree of the district is still in the circle of failure zone, plastic zone extended range to from the middle part of the top plate extension to the surrounding. When the working face by $280 \mathrm{~m}$ advance to $360 \mathrm{~m}$ process, changing the face long mining field of alien mutation effects appear gradually and mining stress distribution is not balanced, the deviatoric stress in roof rock, the rock tensile induced crack and shear yield destructive enhanced, rock mass structure, old or new change, local surrounding rock of irregular broken seriously, plastic zone distribution in abnormal expansion and dynamic growth stage, expansion space from the shallow to the deep, damage range from the lower part extends upwards, the plastic zone shape from round development is the knife shaped, showing time and space under the condition of gradual failure characteristics. The gradual expansion of the plastic damage zone directly leads to the development of the cracks in surrounding rock, and the instability of the layer and the fracture of mining rock.

Comprehensive analysis shows that the development and expansion of the plastic zone of surrounding rock and the expansion of stope roof with variable length can run through the whole process of the coal mining activity. Surrounding rock plastic zone formation and the scope of the development size, failure shape and extended depth or corresponding steady-state adjustment and sexuality changes according to the inherent law of overburden rock activity, so as to provide quantitative criteria for stability evaluation of surrounding rock in different mining stage. 


\section{CONCLUSIONS}

Based on the elasto mechanical properties of limit equilibrium theory, deduced surrounding rock plastic zone radius, shear force mechanical formula for the tangential stress and radial stress, and draws a conclusion that the stability of surrounding rock and the original rock should force, cohesion and internal friction angle is closely related, the shear stress and radial should force and cohesion was positively related to increase with the increase of cohesion; plastic zone radius and the cohesion and internal friction angle is negative correlation, with the cohesion and internal friction angle increases and decreases. Using numerical simulation software to study the forming process, development trend and failure space of the plastic zone of the roof surrounding rock with variable face length, reveals the spatial and temporal expansion effect of the plastic zone gradual destruction of the roof rock.Facet mining roof surrounding rock plastic zone formation produced at the initial stage of plastic failure, the development stage of the formation of elliptical partial plastic ring, extended late in the formation of "cyclic damage ring". Large mining roof surrounding rock plastic zone expansion of space from the shallow to the deep extension transfer, damage range from the lower part to upper part of the vertical extension, the plastic zone shape by circular circle development is the knife shaped and exhibited the gradual failure characteristics under the time and space condition.

\section{ACKNOWLEDGMENT}

This work is supported by Hunan Key Laboratory of Geomechanics and Engineering Safety(Grant No.16GES10) and Xiangtan University Natural Science Foundation(Grant No. 15XZX41).

\section{REFERENCES}

[1] WANG Xinfeng,GAO Mingzhong. Mechanical model of fracture mechanism of stope roof for working face with variable length[J].Journal of China University of Mining and Technology, 2015,44(1):36-45(in Chinese).

[2] GAO Mingzhong, WANG Xinfeng. Numerical simulation analysis of overburden failure in working face of unequal length[J].Journal of Anhui University of Science and Technology(Natural Science),2013,33(4):3236(in Chinese).

[3] CHEN Liwe i,PENG Jianbing, FAN Wen, et al. Analysis of surrounding rock mass plastic zone of round tunnel under non-uniform stress field based on the unified strength theory[J]. Journal of China Coal Society,2007,32(1):20-23(in Chinese).

[4] MA Nianjie,Li Ji,ZHAO Zhiqiang. Distribution of the deviatoric stress field and plastic zone in circular roadway surrounding rock[J].Journal of China University of Mining and Technology, 2015,44(2):206-213(in Chinese).

[5] ZHANG Changguang, FAN Wen, ZHAO Junhai. New solutions of rock plastic displacement and ground response curve for a deep circular tunnel and parametric analysis[J]. Rock and Soil Mechanics,2016,37(1):

[6] 12-24(in Chinese). IANG Minggao,SHI Pingwu, XU Jialin. Mining Pressure and Strata Control[M]. Xuzhou: China University of Mining and Technology press(in Chinese). 